\title{
INTERAÇÃO DO LAPACHOL COM NANOPLATAFORMAS MICELARES DRUG DELIVERY
}

\section{Lapachol interaction with drug delivery micellar nanoplatform}

Katieli da Silva Souza Campanholi ${ }^{1}$, Flávia Amanda Pedroso de Morais ${ }^{1}$, Évelin Lemos de Oliveira ${ }^{1}$, Maycon Renan Santos Lima ${ }^{1}$, Elza Aparecida da Silva ${ }^{2}$, Expedito Leite Silva ${ }^{1}$, Wilker Caetano ${ }^{1}$

${ }^{1}$ Universidade Estadual de Maringá -UEM, Maringá, PR. ${ }^{2}$ União das Escolas Superiores de Rondônia - UNIRON, Departamento de Enfermagem, RO

E-mail: katieli_souza@hotmail.com

RESUMO - A elevada toxicidade de diversos fármacos administrados de forma sistêmica é devido à baixa especificidade às células doentes, resultando em efeitos adversos e desconfortos aos pacientes. Uma estratégia promissora para ampliação da eficácia terapêutica e uso clínico dos princípios ativos é sua incorporação em sistemas micelares nanoestruturados, os quais são amplamente abordados na literatura como drug delivery system. Dessa forma, este artigo contempla aspectos relacionados a capacidade de interação do fármaco natural lapachol com dois sistemas micelares (F127 e P123), visando a obtenção de um novo medicamento para administração sistêmica. Para isso, estudos prévios foram conduzidos para avaliação das variações que a agregação do lapachol resulta em seu comportamento espectral. Posteriormente, a interação do lapachol com os sistemas drug delivery foi investigada pelo monitoramento dos espectros de emissão de fluorescência do fármaco. De uma forma geral, os resultados obtidos foram promissores e enriquecem 0 arsenal terapêutico atual com uma nova proposta de medicamento, cujo fármaco tem reconhecimento etnofarmacológico e científico consagrado.

Palavras-chave: Lapachol, Pluronic ${ }^{\circledR}$, Naftoquinona, Constante de ligação.

ABSTRACT - The high toxicity of several systemically administered drugs is due to low specificity to diseased cells. This results in adverse effects and discomfort to patients. A promising strategy to enhance the therapeutic efficacy and clinical use of active ingredients is their incorporation into nanostructured micellar systems. These nanocarriers are widely addressed in the literature as drug delivery systems. Thus, this paper discusses aspects related to the interaction capacity of the natural drug lapachol with two micellar systems (F127 and P123), aiming to obtain a new drug for systemic administration. For this, previous studies were conducted to evaluate the variations that the aggregation of lapachol results in its spectral behavior. Subsequently, the interaction of lapachol with drug delivery systems was investigated by monitoring the fluorescence emission spectra of the drug. In general, the results obtained were promising and enrich the current therapeutic arsenal with a new medicine proposal, whose drug has established ethnopharmacological and scientific recognition.

Keywords: Lapachol, Poloxamer ${ }^{\circledR}$, Naphthoquinone, Binding constant. 


\section{INTRODUÇÃO}

O lapachol (LP) é uma Naftoquinona (NFq) bioativa que pode ser extraída da serragem de árvores de Ipê roxo, facilmente encontradas no norte e nordeste brasileiro (Hussain et al., 2007). O estudo deste composto desperta o interesse das indústrias farmacêuticas devido a uma série de atividades biológicas, como: efeitos antitumorais, leishmanicidas, bactericidas, malaricidas, inibidores do HIV-1, antiinflamatórios, tripanocidas, antifúngicos, contra larvas Aedes aegypti, entre outras ((Brandão et al., 2018; El-Hawary et al., 2021; Medeiros et al., 2010; Pereira et al., 2006; Pertino et al., 2020). Além disso, estudos in vitro $e$ in vivo têm reportado seu acentuado potencial como inibidor de metástase (Maeda et al., 2008; Mendes Miranda et al., 2021). O uso do Ipê roxo está presente na medicina popular, sendo administrado comumente na forma de chás feitos por infusão das cascas secas e pulverizadas para o tratamento de adenocarcinoma, câncer de esôfago, intestino, pulmões, cabeça, próstata, doença de Hodgkin, leucemia, lúpus e infecções de natureza parasitárias, incluindo a malária (Brandão et al., 2018). Amparados nos benefícios terapêuticos, atualmente o laboratório Farmacêutico do Estado de Pernambuco (LAFEPE) comercializa cápsulas gelatinosas contendo 250 mg de lapachol como auxílio no tratamento do câncer.

Embora o lapachol tenha benefícios consolidados, seu pequeno tamanho molecular pode favorecer a passagem indesejada pelo endotélio (Vieira and Gamarra, 2016). Como consequência, ocorre toxicidade tanto em células sadias quanto nas doentes, acarretando em efeitos colaterais nos pacientes e requerendo maiores frequências de administração do medicamento para a manutenção da concentração terapêutica (Rao et al., 1968). Uma estratégia que tem reduzido os efeitos adversos é a incorporação dos fármacos em sistemas de entrega, amplamente chamados de drug delivery systems. Após a encapsulação do fármaco em matrizes de liberação, as dimensões dos sistemas tornam-se inapropriadas para atravessar as paredes dos vasos de regiões sadias do organismo. No entanto, sabe-se que as regiões acometidas por inflamações ou áreas tumorais têm as células endoteliais com microvasculatura descontínua, o que torna facilitada a entrada e retenção (atividade linfática ineficiente) de nanoplataformas contendo o princípio ativo. Essa é uma estratégia de vetorização passiva amplamente reportada na literatura e conhecida como efeito de permeabilidade e retenção aumentada (efeito EPR; do inglês enhanced permeabilility and retention effect) (Dotivo et al., 2021).

Como sistemas drug delivery, as micelas poliméricas da classe dos Pluronic , constituídas pelos copolímeros tribloco F127 e P123, recebem destaque (Combuca da Silva Junior et al., 2019; da Silva Souza Campanholi et al., 2020). A mínima toxicidade sistêmica, maior tempo de permanência no sistema circulatório, distribuição estreita de tamanho e maior estabilidade do fármaco incorporado tornam esses sistemas protagonistas em publicações científicas em todo mundo (da Silva Souza Campanholi et al., 2020; George, 2011; Gerola et al., 2017; Zarrintaj et al., 2020). Esses drug delivery systems são constituídos por arranjos de poli(óxido de etileno) (POE) e poli(óxido de propileno) (POP), organizados em uma estrutura tribloco do tipo POE-POP-POE (Fig. 1) (Agafonov et al., 2019).

Figura 1. Representação da incorporação do lapachol na micela copolimérica.

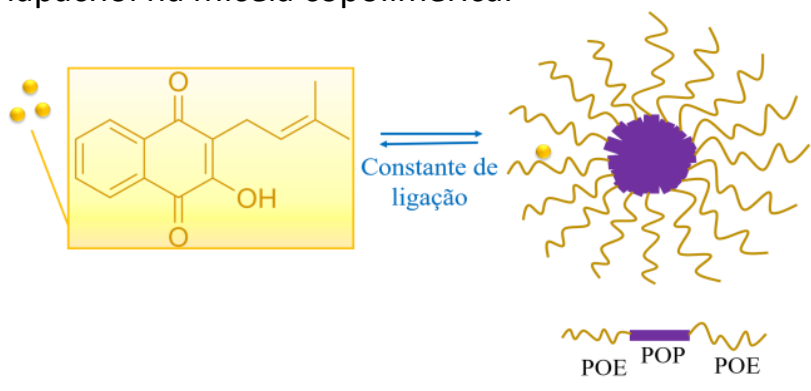

Em meio aquoso e em concentrações relativamente baixas, as cadeias dos copolímeros em bloco existem na forma de unímeros. Entretanto, em condições superiores a temperatura micelar crítica (TMC) e concentração micelar crítica (CMC) esses sistemas se organizam e formam micelas nanoestruturadas, compostas por microdomínios hidrofílicos (corona) e hidrofóbicos (core) (Fig. 2) (da Silva Souza Campanholi et al., 2020). 
Figura 2. Representação das micelas de F127 e P123 e processo de micelização.

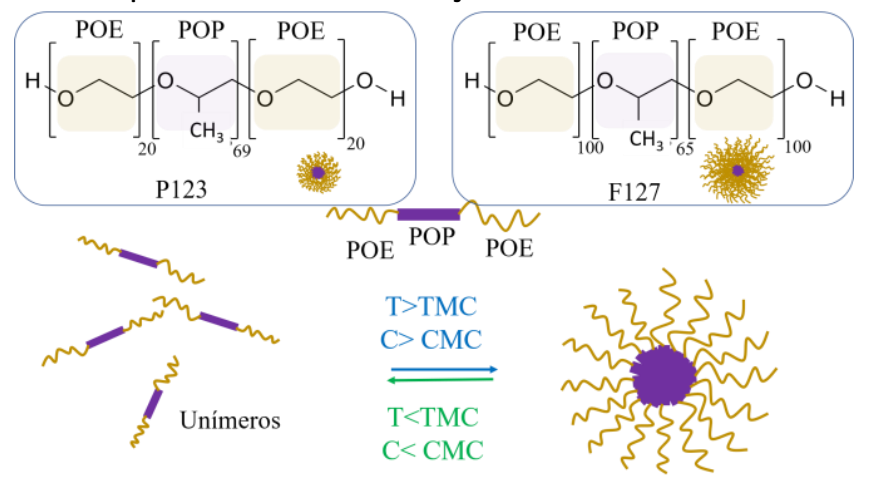

Sua organização estrutural é uma propriedade particular que confere a esses drug delivery systems acentuada capacidade de estabilizar fármacos de diversas naturezas químicas (Campanholi et al., 2018).

Dessa forma, considerando os benefícios terapêuticos amplamente relatados para 0 lapachol, o presente trabalho aborda a capacidade de incorporação deste fármaco em nanoplataformas drug delivery compostas por micelas de F127 e P123, visando à obtenção de uma formulação biomédica (medicamento) estável e promissora para futuros testes in vitro e in vivo.

\section{DESENVOLVIMENTO}

O lapachol $\left(\mathrm{C}_{15} \mathrm{H}_{14} \mathrm{O}_{3}\right.$, massa molar 242,27 g.mol ${ }^{-1}$ ) foi extraído a partir da serragem do Ipê roxo (Tabebuia impetiginosa (Mart. ex DC.) Standl.) de madeira obtida no Campus da Universidade Estadual de Maringá, Paraná, Brasil. A extração foi realizada com solução saturada de carbonato de sódio e agitação/maceração periódica durante aproximadamente $1 \mathrm{~h}$. Logo após, a suspensão foi filtrada e o filtrado acidificado com ácido clorídrico $6 \mathrm{~mol}^{-\mathrm{L}^{-1}}$. $\mathrm{O}$ sólido amarelo obtido passou por filtração a vácuo, lavagem com água destilada a baixas temperaturas e recristalização com etanol. $\mathrm{O}$ rendimento médio obtido foi de $1 \%(\mathrm{~m} / \mathrm{m})$. A caracterização do lapachol foi realizada por ${ }^{1} \mathrm{H}$ NMR (Varian, espectrómetro Gemini, $300 \mathrm{MHz}$ ) e ${ }^{13} \mathrm{C}$ NMR $(75,45 \mathrm{MHz})$ em clorofórmio deuterado $\left(\mathrm{CDCl}_{3}\right)$, sendo os resultados concordantes com a literatura. Detalhamentos do processo de extração e caracterização foram fornecidos em publicação anterior (Campanholi et al., 2018).

Os copolímeros micelares Pluronic ${ }^{\circledR}$ P123 (massa molar $5.800 \mathrm{~g} \mathrm{~mol}^{-1}$ ) e F127 (massa molar 12.600 g. $\mathrm{mol}^{-1}$ ) foram comprados da SigmaAldrich. Todo etanol utilizado foi previamente purificado por destilação à pressão atmosférica e posteriormente armazenamento em peneira molecular de $3 \AA ̊$ ativada.

\section{Estudo do comportamento agregacional do lapachol}

Os estudos de agregação foram realizados de forma prévia para identificar as propriedades espectroscópicas (via absorção eletrônica UV-Vis e emissão de fluorescência) do estado monomérico (solubilizado) ou autoagregado do lapachol (aglomerado de moléculas da NFq). Para isso, misturas dos solventes foram preparadas nas composições $0,20,40,50,60,70,80,90$ e $100 \%(v / v)$ de água em etanol. Para cada composição de solvente, foi adicionado lapachol suficiente para obter a concentração de $4,6 \times 10^{-5}$ mol. $L^{-1}$ para os estudos monitorados por absorção eletrônica UV-Vis e $5 \times 10^{-6} \mathrm{~mol}^{-L^{-1}}$ para obtenção de espectros de emissão de fluorescência. Os espectros foram coletados após 15 minutos de estabilização do preparo da solução, a $30,0{ }^{\circ} \mathrm{C}$ (Vilsinski et al., 2015). As informações espectrais coletadas servirão como ferramenta para posterior compreensão do comportamento do lapachol na presença das micelas de F127 e P123.

\section{Interação do Lapachol com micelas de F127 e P123}

O experimento foi conduzido a partir de uma solução de lapachol na concentração $2 \times 10^{-6}$ mol. $\mathrm{L}^{-1}$ preparada em meio aquoso, com $\mathrm{pH} 7,0$. Essa solução foi adicionada a uma cubeta de quartzo com as quatro faces polidas, onde recebeu continuamente pequenas alíquotas de solução aquosa do copolímero micelar F127, preparada na concentração $4 \%(\mathrm{~m} / \mathrm{v})$. A cada adição de solução de copolímero, espectros de absorção eletrônica UV-Vis e emissão de fluorescência foram coletados na temperatura de $30,0^{\circ} \mathrm{C}$. Posteriormente, o máximo de emissão de fluorescência do lapachol foi plotado em função da concentração do copolímero F127 para ajuste da isoterma obtida. O mesmo procedimento foi realizado utilizando uma nova solução de lapachol na mesma concentração e uma solução aquosa micelar de P123 4\% (m/v) para as adições sequenciais de copolímero. $O$ valor da constante de ligação foi determinado a partir da Eq. 1 (Caetano et al., 2000). 


$$
F=F_{f}+\frac{\left(F_{0}+F_{f}\right)}{\left(\frac{1}{K_{b}([S]-C M C)^{n}}\right)+1}
$$

Onde $F$ corresponde à intensidade de fluorescência, $F f$ emissão da $\mathrm{NFq}$ ligada ao surfactante, $F_{o}$ a emissão na ausência de surfactante, [S] a concentração de surfactante, CMC a concentração micelar crítica e $n$ o número de molécula de lapachol por micelas copoliméricas.

Os estudos de interação do lapachol com os sistemas micelares F127 e P123 foram realizados em dois tempos. Com o objetivo de avaliar a interação primária lapachol-micela, medidas foram obtidas logo após a adição dos copolímeros F127 e P123. Estudos complementares foram realizados com medidas coletadas após $48 \mathrm{~h}$, visando conhecer o comportamento lapachol-micela após a estabilização termodinâmica. Nessa situação foram preparadas soluções individuais de lapachol em para cada adição/concentração do copolímero micelar. As soluções foram mantidas em repouso, na temperatura ambiente, durante 48 h. Posteriormente, as análises espectroscópicas de cada solução foram realizadas.

\section{RESULTADOS E DISCUSSÃO}

$O$ efeito da polaridade do solvente pode causar diversas mudanças no comportamento de absorção dos compostos, podendo atuar como uma importante ferramenta para compreensão da dinâmica estrutural do fármaco quando exposto a ambientes fisiológicos (Etienne et al., 2014; Kiani et al., 2020). Para esse contexto, avaliações envolvendo misturas binárias água e etanol são recorrentes na literatura e atuam na identificação de possíveis formas autoagregadas dos fármacos. Espécies autoagregadas são formadas quando interações não covalentes fármaco-fármaco superam as forças de interação fármaco-solvente, levando a baixa solubilidade e possíveis precipitados. Esses estudos são fundamentais para posterior comparação e elucidação dos fenômenos de monomerização ou autoagregação que podem ocorrer após a incorporação do lapachol nos sistemas micelares de F127 e P123 (Murakami, 2002; Vilsinski et al., 2015). Os espectros de absorção eletrônica e emissão de fluorescência do lapachol nas diferentes porcentagens água/etanol (v/v) são apresentados na Fig. 3.
Figura 3. (A) Espectros de absorção eletrônica do lapachol $\left(4,6 \times 10^{-5} \mathrm{~mol}^{-\mathrm{L}^{-1}}\right)$ variando a proporção de etanol e água, (B) $\lambda_{\max }$ de absorção, (C) espectros de emissão de fluorescência do lapachol $\left(5,0 \times 10^{-6} \mathrm{~mol} . \mathrm{L}^{-1}\right)$ variando a proporção água e etanol, e (D) $\lambda_{\max }$ de emissão. Os espectros de emissão foram obtidos com $\lambda_{\text {exc }}=225 \mathrm{~nm}$, fenda 10/10 nm. A seta indica a direção da variação espectral com o aumento do teor de água no sistema.
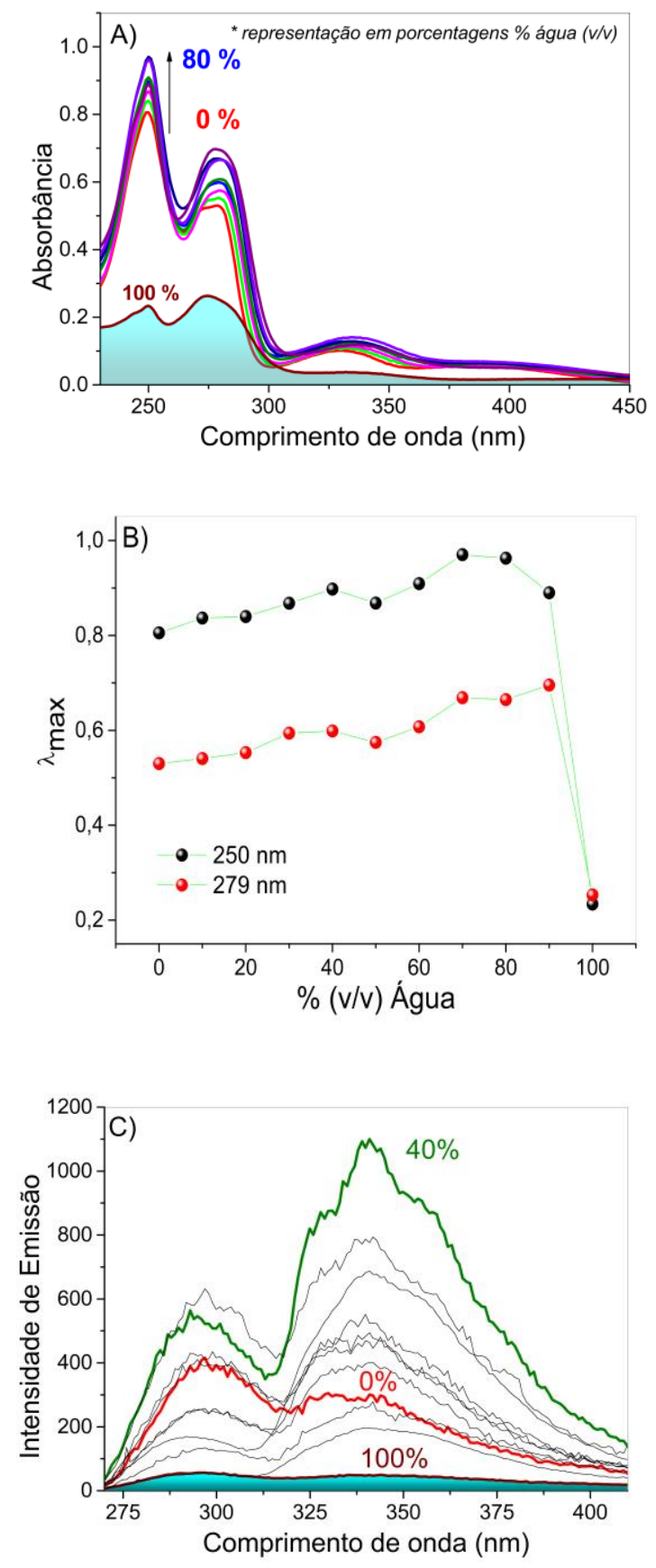


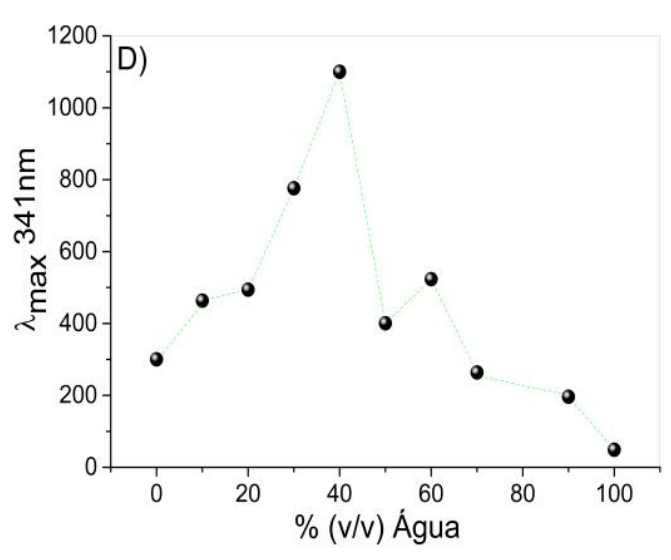

Pode-se verificar a partir dos espectros de absorção eletrônica UV-Vis do lapachol (Fig. 3A) a ausência de deslocamentos significativos nos comprimentos de onda de máxima absorção com o aumento da polaridade do meio. Em termos de intensidades de absorção, notou-se um aumento contínuo acompanhado de alargamento das principais bandas até aproximadamente $80 \%(\mathrm{v} / \mathrm{v})$ de água (Fig. 3B). Igualmente, os espectros de emissão de fluorescência nestes sistemas (Fig. 3C-D) mostraram um aumento nas intensidades na região de 290 e $340 \mathrm{~nm}$, mais pronunciado até $40 \%(\mathrm{v} / \mathrm{v})$ de água. Essas variações espectroscópicas (Fig. 3A-D), dependentes das proporções de água e etanol, podem estar vinculadas ao processo de solvatação preferencial, que considera a presença de três espécies na mistura binária de solventes (água, etanol e clusters, compostos pela associação água-etanol) ((Delgado et al., 2011; Farajtabar et al., 2011; Pal et al., 2021). Dados referentes a sistemas mistos água-álcool e solvatação preferencial foram anteriormente preconizados na literatura e avaliados em termos das interações soluto-solvente e solvente-solvente (Farajtabar et al., 2011). Nesse aspecto, o aumento contínuo da absorbância da NFq até a proporção de $80 \%(\mathrm{v} / \mathrm{v})$ de água (Fig. 3A-B) sugere diferenças entre a composição da camada de solvatação e o seio da solução. Nessa condição, o lapachol, acomodado em uma esfera de solvatação particular, pode ser solvatado por efeitos de interações não específicos (interação preferencial com o componente de maior constante dielétrica "enriquecimento dielétrico") e interações específicas, como ligações de hidrogênio (Ghoneim, 2001; Józefowicz, 2011). Tratando-se ainda da Fig. 3A, pode-se notar uma redução do máximo de absorção do lapachol em
$250 \mathrm{~nm}$ e um aumento da intensidade em 279 $\mathrm{nm}$ entre 80 e $100 \%(\mathrm{v} / \mathrm{v})$ de água. Esse comportamento pode estar associado a efeitos agregacionais e a pequenas variações de $\mathrm{pH}$ no sistema, que resulta em mudanças na proporção relativa das formas protolíticas da espécie (pKa do lapachol próximo a 6,2) (Campanholi et al., 2018). A porcentagem de água agregacional crítica para o lapachol (porcentagem limite em que não se verifica autoagregados) (Gracetto et al., 2010), encontra-se na região de $80 \%(\mathrm{v} / \mathrm{v})$ de água.

Após compreender o comportamento do composto em meios concentrados em água, estudou-se a interação primária da NFq com os copolímeros micelares, sistemas nanoestruturados biocompatíveis que objetivam garantir ao lapachol maior estabilidade e biodisponibilidade em administrações intravenosas (Rangel-Yagui et al., 2005; Vieira and Gamarra, 2016). A afinidade da NFq com os Pluronic (Fig. 1) foi estimada pela constante de ligação $\left(K_{b}\right)$, parâmetro obtido a partir de ajustes que consideram a variação na intensidade de emissão em função da concentração do copolímero micelar, considerando os parâmetros $\mathrm{CMC}$ (concentração micelar crítica) e $n$ (número de micelas por moléculas de NFq) (Caetano and Tabak, 1999; Pellosi et al., 2013) (Eq. 1). Os espectros de emissão de fluorescência do lapachol encontram-se apresentados no Fig. 4.

Figura 4. (A) Espectros de emissão de fluorescência do lapachol $\left(2,0 \mu \mathrm{mol} \mathrm{L^{-1 }}\right)$ com a adição de alíquotas de solução com micelas de P123 e F127, (B-C) máximos de emissão de fluorescência em função da concentração de copolímero micelar adicionado. Espectros obtidos usando $\lambda_{\text {exc }}=230 \mathrm{~nm}$ e fenda $10 / 10 \mathrm{~nm}$ ).

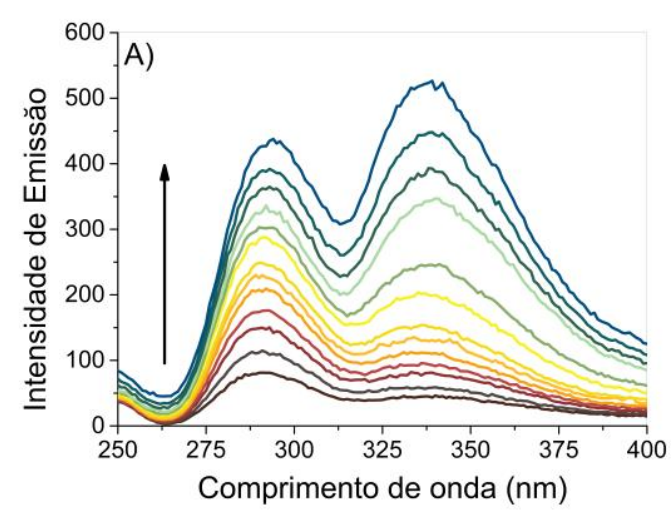



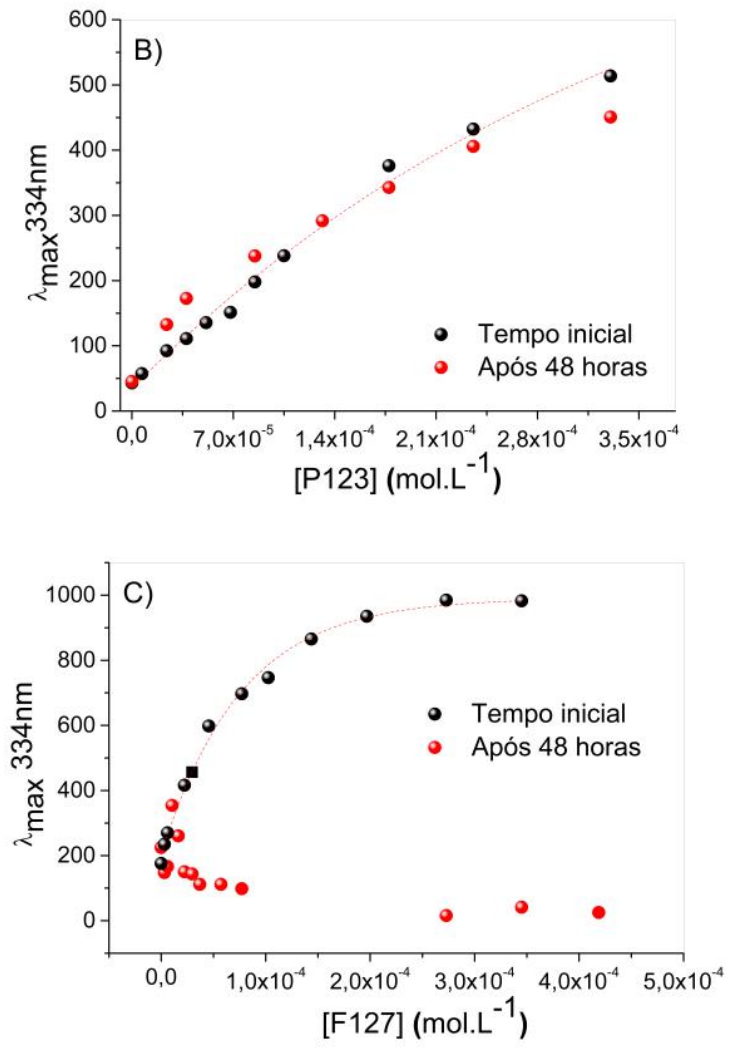

Com base na Fig. 4A pode-se verificar baixa intensidade de emissão de fluorescência do lapachol em meio aquoso, fato justificado pelo efeito da autoagregação e processos de supressão de fluorescência por moléculas de água (Gracetto et al., 2010; Kyrychenko et al., 2011; Lakowicz, 2006; Lopez Arbeloa et al., 1993). $A$ adição subsequente de alíquotas do Pluronic $^{\circledR}$ permitiu um aumento nas intensidades de emissão da NFq no tempo inicial (Fig. 4A-C). Esse fato pode ser associado a redução da supressão por moléculas de água concomitante a efeitos de monomerização decorrentes da partição da NFq para microambientes micelares, que levam à recuperação da fluorescência (Alexandridis and Alan Hatton, 1995). A semelhança entre as isotermas de ligação após a estabilização termodinâmica (48 h) mostrada na Fig. 4B reporta a ausência de efeitos temporais consideráveis de redistribuição da $\mathrm{NFq}$ com o passar do tempo (Vilsinski et al., 2015). Para o sistema obtido em F127 (Fig. 4C) verificou-se efeitos similares, os quais demonstraram associação imediata à micela propiciando a desagregação e redistribuição no microambiente micelar durante um processo primário de ligação. Entretanto, as medidas após $48 \mathrm{~h}$ mostraram processos de reorganização estrutural da NFq no microambiente do nanocarreador F127 (Vilsinski et al., 2015). Os dados de absorção eletrônica UVVis para o lapachol em F127 após 48 h corroboraram com a hipótese de agregação, evidenciado pela diminuição e alargamento das principais bandas de absorção eletrônica (dados não mostrados).

Os valores de $K_{b}$ refletem a interação da $\mathrm{NFq}$ com as nanoplataformas de liberação (Tab. 1). Os dados foram obtidos considerando a Eq. 1 para as Figuras 4B-C no tempo inicial. As isotermas no tempo de equilíbrio não puderam ser ajustadas devido à complexidade do perfil de interação (Hosseinzadeh et al., 2008; Moore et al., 2007; Vilsinski, 2013). Os valores de $K_{b}$ considerados foram aqueles que ofereceram a melhor qualidade do gráfico de resíduos regular obtido nos ajustes não lineares.

Tabela 1. Valores de $K_{b}$ do lapachol com sistemas micelares P123 e F127 no tempo inicial.

\begin{tabular}{cll}
\hline & LP/F127 & LP/P123 \\
$\mathrm{K}_{\mathrm{b}}\left(10^{3}{\left.\mathrm{~L} . \mathrm{mol}^{-1}\right)}^{-1}\right.$ & $16,80 \pm 0,80$ & $1,28 \pm 0,03$ \\
$\mathrm{CMC}\left(10^{-6} \mathrm{~mol}^{-1}\right)$ & $4,41 \pm 0,90$ & $29,60 \pm 3,90$ \\
$N$ & $1,000 \pm 0,001$ & $1,000 \pm 0,001$ \\
\hline
\end{tabular}

Segundo os dados apresentados na Tab. 1 pode-se verificar maior interação do lapachol com o copolímero F127 no tempo inicial, uma vez que para esse sistema foi obtido maior valor de $\mathrm{K}_{\mathrm{b}}$. Sabe-se que micelas de F127 apresentam o segmento POE com maior extensão (Fig. 2) comparadas às micelas P123 (Campanholi et al., 2018). Dessa forma, regido por seu caráter anfifílico (Campanholi et al., 2018), o lapachol pode ter encontrado um ambiente suscetível para acomodação e estabilização inicial na porção hidrofílica das micelas de F127. Os valores de $n$ (Tab. 1) foram próximos da unidade e referem-se ao número de lapachol por micela, indicando a presença de aproximadamente uma molécula de fármaco solubilizada por micela copolimérica. Os valores de CMC verificados (Tab. 1) foram razoáveis, tanto para o F127 (CMC literatura na ordem de $10^{-5}$ ) quanto para o P123 (CMC literatura na ordem de $10^{-6}$ ) (Alexandridis and Alan Hatton, 1995; Su et al., 2002).

De uma forma geral, os estudos de ligação do lapachol com os sistemas nanoestruturados F127 e P123 mostraram viabilidade na obtenção do sistema lapacholmicela. Essa é uma situação que pode trazer grandes benefícios terapêuticos e melhor aproveitamento das propriedades do fármaco, 
uma vez que os efeitos de toxicidade tendem a ser reduzidos (Vieira and Gamarra, 2016).

\section{CONCLUSÃO}

A solubilidade do lapachol mostrou-se depende da polaridade do meio, sendo a porcentagem de água agregacional crítica próxima de $80 \%(\mathrm{v} / \mathrm{v})$. Tanto as micelas de F127 quanto P123 foram capazes de incorporar e estabilizar o lapachol, condição esta que pode trazer uma série de benefícios terapêuticos. Amparados nos dados obtidos e no conhecimento etnofarmacológico e científico consagrado do fármaco, pode-se considerar os sistemas nanoestruturados F127/Lapachol e P123/Lapachol como potenciais medicamentos para futuros estudos in vitro e in vivo.

\section{REFERÊNCIAS}

Agafonov, M., Volkova, T., Kumeev, R., Chibunova, E., Terekhova, I., 2019. Impact of pluronic F127 on aqueous solubility and membrane permeability of antirheumatic compounds of different structure and polarity. J. Mol. Liq. 274, 770-777. https://doi.org/10.1016/j.molliq.2018.11.060

Alexandridis, P., Alan Hatton, T., 1995. Poly(ethylene oxide)-poly(propylene oxide)poly(ethylene oxide) block copolymer surfactants in aqueous solutions and at interfaces: thermodynamics, structure, dynamics, and modeling. Colloids Surfaces A Physicochem. Eng. Asp. 96, 1-46. https://doi.org/10.1016/0927-7757(94)03028-X

Brandão, G.C., Rocha Missias, F.C., Arantes, L.M., Soares, L.F., Roy, K.K., Doerksen, R.J., Braga de Oliveira, A., Pereira, G.R., 2018. Antimalarial naphthoquinones. Synthesis via click chemistry, in vitro activity, docking to Pf DHODH and SAR of lapachol-based compounds. Eur. J. Med. Chem. 145, 191-205. https://doi.org/10.1016/j.ejmech.2017.12.051

Caetano, W., Tabak, M., 1999. Interaction of chlorpromazine and trifluoperazine with ionic micelles: electronic absorption spectroscopy studies. Spectrochim. Acta Part A Mol. Biomol. Spectrosc. 55, 2513-2528. https://doi.org/10.1016/S1386-1425(99)00043-8

Caetano, W., Tabak, M., S, U. De, 2000. Interaction of Chlorpromazine and
Trifluoperazine with Anionic Sodium Dodecyl Sulfate (SDS ) Micelles: Electronic Absorption and Fluorescence Studies 81, 69-81. https://doi.org/10.1006/jcis.2000.6720

Campanholi, K. da S.S., Gerola, A.P., Vilsinski, B.H., de Oliveira, É.L., de Morais, F.A.P., Rabello, B.R., Braga, G., Calori, I.R., Silva, E.L., Hioka, N., Caetano, W., 2018. Development of Pluronic ${ }^{\circledR}$ nanocarriers comprising Pheophorbide, ZnPheophorbide, lapachol and $\beta$-lapachone combined drugs: Photophysical and spectroscopic studies. Dye. Pigment. https://doi.org/10.1016/i.dyepig.2018.04.057

Combuca da Silva Junior, R., da Silva Souza Campanholi, K., Pedroso de Morais, F.A., Soares dos Santos Pozza, M., Tadeu dos Santos, G., Hioka, N., Caetano, W., 2019. Development and applications of safranine-loaded Pluronic ${ }^{\circledR}$ F127 and P123 photoactive nanocarriers for prevention of bovine mastitis: In vitro and in vivo studies. Dye. Pigment. 167, 204-215. https://doi.org/10.1016/i.dyepig.2019.04.037

da Silva Souza Campanholi, K., Jaski, J.M., da Silva Junior, R.C., Zanqui, A.B., Lazarin-Bidóia, D., da Silva, C.M., da Silva, E.A., Hioka, N., Nakamura, C.V., Cardozo-Filho, L., Caetano, W., 2020. Photodamage on Staphylococcus aureus by natural extract from Tetragonia tetragonoides (Pall.) Kuntze: Clean method of extraction, characterization and photophysical studies. J. Photochem. Photobiol. B Biol. https://doi.org/10.1016/i.jphotobiol.2019.11176 $\underline{3}$

Delgado, D.R., Holguín, A.R., Almanza, O.A., Martínez, F., Marcus, Y., 2011. Solubility and preferential solvation of meloxicam in ethanol+water mixtures. Fluid Phase Equilib. 305, 88-95.

https://doi.org/10.1016/i.fluid.2011.03.012

Dotivo, N.C., Rezende, R.P., Pessoa, T.B.A., Salay, L.C., Huachaca, N.S.M., Romano, C.C., Marques, E. de L.S., Costa, M.S., de Moura, S.R., Pirovani, C.P., Dias, J.C.T., 2021. Immobilization of PR4A3 enzyme in pluronic F127 polymeric micelles against colorectal adenocarcinoma cells and increase of in vitro bioavailability. Int. J. Biol. Macromol. 166, 1238-1245. https://doi.org/10.1016/j.ijbiomac.2020.11.006 
El-Hawary, S.S., Taher, M.A., Amin, E., Fekry AbouZid, S., Mohammed, R., 2021. Genus Tabebuia: A comprehensive review journey from past achievements to future perspectives. Arab. J. Chem. 14, 103046. https://doi.org/10.1016/j.arabjc.2021.103046

Etienne, T., Michaux, C., Monari, A., Assfeld, X., Perpète, E. a., 2014. Theoretical computation of Betain B30 solvatochromism using a Polarizable Continuum Model. Dye. Pigment. 100, 24-31. https://doi.org/10.1016/j.dyepig.2013.07.017

Farajtabar, A., Jaberi, F., Gharib, F., 2011. Preferential solvation and solvation shell composition of free base and protonated 5, 10, 15, 20-tetrakis(4-sulfonatophenyl)porphyrin in aqueous organic mixed solvents. Spectrochim. Acta. A. Mol. Biomol. Spectrosc. 83, 213-20. https://doi.org/10.1016/j.saa.2011.08.020

George, A., 2011. Advances in biomimetics. pp. 251-253. https://doi.org/10.5772/574

Gerola, A.P., de Morais, F.A.P., Costa, P.F.A., Kimura, E., Caetano, W., Hioka, N., 2017. Characterization of chlorophyll derivatives in micelles of polymeric surfactants aiming photodynamic applications. Spectrochim. Acta Part A Mol. Biomol. Spectrosc. 173, 213-221. https://doi.org/10.1016/j.saa.2016.09.019

Ghoneim, N., 2001. Study of the preferential solvation of some betaine dyes in binary solvent mixtures. Spectrochim. Acta. A. Mol. Biomol. Spectrosc. 57, 1877-84. https://doi.org/10.1016/S1386-1425(01)00418-8

Gracetto, A.C., Batistela, V.R., Caetano, W., Oliveira, H.P.M. de, Santos, W.G., Cavalheiro, C.C.S., Hioka, N., 2010. Unusual 1,6-diphenyl1,3,5-hexatriene (DPH) spectrophotometric behavior in water/ethanol and water/DMSO mixtures. J. Braz. Chem. Soc. 21, 1497-1502. https://doi.org/10.1590/S0103-

$\underline{50532010000800013}$

Hosseinzadeh, R., Maleki, R., Matin, A.A., Nikkhahi, Y., 2008. Spectrophotometric study of anionic azo-dye light yellow (X6G) interaction with surfactants and its micellar solubilization in cationic surfactant micelles. Spectrochim. Acta. A. Mol. Biomol. Spectrosc. 69, 1183-7. https://doi.org/10.1016/i.saa.2007.06.022
Hussain, H., Krohn, K., Ahmad, U., Miana, A., 2007. Lapachol: an overview 2007, 145-171. https://doi.org/10.3998/ark.5550190.0008.204

Józefowicz, M., 2011. The influence of hydrogen bonds and preferential solvation on spectroscopic properties of methyl pdimethylaminobenzoate and its ortho derivative in binary solvent mixture. Chem. Phys. 383, 1926.

https://doi.org/10.1016/j.chemphys.2011.03.025

Kiani, M., Ghiasi, R., Pasdar, H., Mirza, B., 2020. Computational investigation of solvent polarity effect on the structure and properties of a (OC) $4 \mathrm{Cr}$-biscarbene complex in the singlet ground state and lowest singlet excited state. J. Mol. Liq. 300, 112327. https://doi.org/10.1016/i.molliq.2019.112327

Kyrychenko, A., Sevriukov, I.Y., Syzova, Z.A., Ladokhin, A.S., Doroshenko, A.O., 2011. Partitioning of 2,6-Bis(1H-Benzimidazol-2yl)pyridine fluorophore into a phospholipid bilayer: complementary use of fluorescence quenching studies and molecular dynamics simulations. Biophys. Chem. 154, 8-17. https://doi.org/10.1016/j.bpc.2010.12.001

Lakowicz, J.R., 2006. Principles of Fluorescence Spectroscopy, $3^{\circ}$ edition. ed. New York. https://doi.org/10.1007/978-0-387-46312-4

Lopez Arbeloa, T., Lopez Arbeloa, F., Tapia, M.J., Lopez Arbeloa, I., 1993. Hydrogen-bonding effect on the photophysical properties of 7aminocoumarin derivatives. J. Phys. Chem. 97, 4704-4707.

https://doi.org/10.1021/j100120a024

Maeda, M., Murakami, M., Takegami, T., Ota, T., 2008. Promotion or suppression of experimental metastasis of B16 melanoma cells after oral administration of lapachol. Toxicol. Appl. Pharmacol. 229, 232-8. https://doi.org/10.1016/i.taap.2008.01.008

Medeiros, C.S., Pontes-Filho, N.T., Camara, C.A., Lima-Filho, J. V, Oliveira, P.C., Lemos, S.A., Leal, A.F.G., Brandão, J.O.C., Neves, R.P., 2010. Antifungal activity of the naphthoquinone betalapachone against disseminated infection with Cryptococcus neoformans var. neoformans in dexamethasone-immunosuppressed Swiss mice. 
Braz. J. Med. Biol. Res. 43, 345-9. https://doi.org/10.1590/S0100$\underline{879 \times 2010007500012}$

Mendes Miranda, S.E., Alcântara Lemos, J. de, Fernandes, R.S., Silva, J. de O., Ottoni, F.M., Townsend, D.M., Rubello, D., Alves, R.J., Cassali, G.D., Ferreira, L.A.M., de Barros, A.L.B., 2021. Enhanced antitumor efficacy of lapachol-loaded nanoemulsion in breast cancer tumor model. Biomed. Pharmacother. 133, 110936. https://doi.org/10.1016/j.biopha.2020.110936

Moore, S.A., Harris, A.A., Palepu, R.M., 2007. Spectroscopic investigations on the binding of ammonium salt of 8-anilino-1-naphthalene sulfonic acid with non-ionic surfactant micelles in aqueous media. Fluid Phase Equilib. 251, 110113. https://doi.org/10.1016/i.fluid.2006.11.009

Murakami, K., 2002. Thermodynamic and kinetic aspects of self-association of dyes in aqueous solution. Dye. Pigment. 53, 31-43. https://doi.org/10.1016/S0143-7208(01)00104-8

Pal, J., Patla, A., Subramanian, R., 2021. Thermodynamic properties of forming methanol-water and ethanol-water clusters at various temperatures and pressures and implications for atmospheric chemistry: A DFT study. Chemosphere 272, 129846. https://doi.org/10.1016/i.chemosphere.2021.129 $\underline{846}$

Pellosi, D.S., Estevão, B.M., Freitas, C.F., Tsubone, T.M., Caetano, W., Hioka, N., 2013. Photophysical properties of erythrosin ester derivatives in ionic and non-ionic micelles. Dye. Pigment. 99, 705-712. https://doi.org/10.1016/j.dyepig.2013.06.026

Pereira, E.M., Machado, T. de B., Leal, I.C.R., Jesus, D.M., Damaso, C.R. de A., Pinto, A.V., Giambiagi-deMarval, M., Kuster, R.M., Santos, K.R.N. dos, 2006. Tabebuia avellanedae naphthoquinones: activity against methicillinresistant staphylococcal strains, cytotoxic activity and in vivo dermal irritability analysis. Ann. Clin. Microbiol. Antimicrob. 5, 5. https://doi.org/10.1186/1476-0711-5-5

Pertino, M.W., F. de la Torre, A., SchmedaHirschmann, G., Vega, C., Rolón, M., Coronel, C., Rojas de Arias, A., Leal López, K., Carranza-
Rosales, P., Viveros Valdez, E., 2020. Synthesis, trypanocidal and anti-leishmania activity of new triazole-lapachol and nor-lapachol hybrids. Bioorg. Chem. 103, 104122. https://doi.org/10.1016/i.bioorg.2020.104122

Rangel-Yagui, C.O., Hsu, H.W.L., Pessoa-Jr, A., Tavares, L.C., 2005. Micellar solubilization of ibuprofen: influence of surfactant head groups on the extent of solubilization. Rev. Bras. Ciências Farm. 41, 237-246. https://doi.org/10.1590/S1516$\underline{93322005000200012}$

Rao, K. V, Mcbride, T.J., Oleson, J.J., 1968. Recognition and Evaluation of Lapachol as an Antitumor Agent and Evaluation of Lapachol as an Antitumor. Cancer Resour. 28, 1952-1954. https://doi.org/Published October 1968

Su, Y., Wang, J., Liu, H., 2002. FTIR Spectroscopic Investigation of Effects of Temperature and Concentration on PEO-PPO-PEO Block Copolymer Properties in Aqueous Solutions. Macromolecules 35, 6426-6431. https://doi.org/10.1021/ma0105284

Vieira, D.B., Gamarra, L.F., 2016. Advances in the use of nanocarriers for cancer diagnosis and treatment. Einstein (São Paulo) 14, 99-103. https://doi.org/10.1590/S1679-45082016RB3475

Vilsinski, B.H., 2013. Estudos das Propriedades Físico-Químicas e Fotodinâmicas da Ftalocianina de Alumínio cloro (AlPcCl) em Sistemas Homogêneos e Encapsulada em Copolímeros

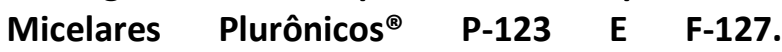
Universidade Estadual de Maringá.

Vilsinski, B.H., Gerola, A.P., Lemos, É.O., Barbosa, P.M., Campanholi, K.S.S., César, G.B., Tessaro, A.L., Hioka, N., Caetano, W., 2015. Spectroscopic study of aluminum phthalocyanine chloride (AIPcCl) in homogeneous and microheterogeneous media consisting of P-123 and F127 polymeric micelles. Quim. Nova 38, 631-639. https://doi.org/10.5935/0100-4042.20150056

Zarrintaj, P., Ramsey, J.D., Samadi, A., Atoufi, Z., Yazdi, M.K., Ganjali, M.R., Amirabad, L.M., Zangene, E., Farokhi, M., Formela, K., Saeb, M.R., Mozafari, M., Thomas, S., 2020. Poloxamer: A versatile tri-block copolymer for biomedical applications. Acta Biomater. 110, 37-67. 
https://doi.org/10.1016/j.actbio.2020.04.028 